\title{
Efficient Memory Page Replacement on Web Server Clusters
}

\author{
Ji Yung Chung and Sungsoo Kim \\ Graduate School of Information and Communication \\ Ajou University, Wonchun-Dong, Paldal-Gu \\ Suwon, Kyunggi-Do, 442-749, Korea \\ \{abback, sskim\}@madang.ajou.ac.kr
}

\begin{abstract}
The concept of network memory was introduced for the efficient exploitation of main memory in a cluster. Network memory can be used to speed up applications that frequently access large amount of disk data. In this paper, we present a memory management algorithm that does not require prior knowledge of access patterns and that is practical to implement under the web server cluster. In addition, our scheme has a good user response time for various access distributions of web documents. Through a detailed simulation, we evaluate the performance of our memory management algorithms.
\end{abstract}

\section{Introduction}

With the growing popularity of the internet, services using the world wide web are increasing. However, the overall increase in traffic on the web causes a disproportionate increase in client requests to popular web sites. Performance and high availability are critical for web sites that receive large numbers of requests $[1,2$, 3].

A cluster is a type of distributed processing system and consists of a collection of interconnected stand-alone computers working together. Cluster systems present not only a low cost but also a flexible alternative to fault tolerant computers for applications that require high throughput and high availability.

Processing power was once a dominant factor in the performance of initial cluster systems. However, as successive generations of hardware appeared, the processor decreased its impact on the overall performance of the system [4]. Now, memory bandwidth has replaced the role of the processor as a performance bottleneck. The impact of networking has also decreased with the 100Mbps ethernet. Thus, efficient memory management is very important for overall cluster system performance.

This work is supported in part by the Ministry of Information \& Communication in Republic of Korea ("University Support Program<2001>” supervised by IITA).

This work is supported in part by the Ministry of Education of Korea (Brain Korea 21 Project supervised by Korea Research Foundation).

P.M.A. Sloot et al. (Eds.): ICCS 2002, LNCS 2331, pp. 1042-1050, 2002.

(C) Springer-Verlag Berlin Heidelberg 2002 
The concept of network memory was introduced for the efficient exploitation of main memory in a cluster. Network memory is the aggregate main memory in the cluster and can be used to speed up applications that frequently access large amounts of disk data.

This paper presents a memory management algorithm that always achieves a good user response time for various access distributions of web documents.

The remainder of the paper is organized as follows. Section 2 presents related work on cluster memory management. In section 3, we explain the clustered web server architecture that is considered in this paper. Section 4 explains our memory management algorithm and section 5 presents simulation results. Finally, section 6 summarizes with concluding remarks.

\section{Related Work}

Recently, some papers on the memory management of the cluster have studied the method of utilizing the idle client's memory [5, 6, 7]. The active client forwards cache entries that overflow its local cache directly to the idle node. The active client can then access this remote cache until the remote node becomes active. However, in these methods, a client's request must go to the disk if the requested block does not exist in the limited memory, even though another node has that block.

The Greedy Forwarding algorithm deals with the memory of the cluster system as a global resource, but the algorithm does not attempt to coordinate the contents of this memory [8]. The main problem with this policy is that global memory is underutilized because of duplication.

Duplicate Elimination [9] takes the other extreme approach. Since it is inexpensive to fetch a duplicate page from remote memory, compared to a disk input/output (I/O), every duplicate page is eliminated before a single page. Each node maintains two LRU (Least Recently Used) lists, one for single pages and the other for duplicate pages. The advantage of Duplicate Elimination is that it has a high global hit rate because of the global memory management. However, a main drawback of Duplicate Elimination is that the local hit rate of some nodes reduces because of the smaller cache size, even if the global hit rate increases.

In order to adjust the duplication rate of the data page dynamically, the $\mathrm{N}$-chance Forwarding algorithm forwards the last copy of the page from one server to a randomly chosen server, $\mathrm{N}$ times, before discarding it from global memory [8].

Also, the Hybrid algorithm dynamically controls the amount of duplication by comparing the expected cost of an LRU single page and an LRU duplicate page [9]. The expected cost is defined as the product of the latency to fetch the page back into memory and a weighting factor that gives a measure of the likelihood of the page being accessed next in memory. The Hybrid algorithm has a good response time on average, but it does not have a minimum response time for a special range of workload and node configuration.

In this paper, we present a memory management algorithm that does not require prior knowledge of access patterns and that is practical to implement under the web 
server cluster. Also, this method always has a good user response time for various access distributions of web documents.

\section{Web Server Cluster Architecture}

In order to handle millions of accesses, a general approach adopted by popular web sites is to preserve one virtual URL (Uniform Resource Locator) interface and use a distributed server architecture that is hidden from the user.

Thus, we consider the architecture of cluster system that consists of the load balancer and a set of document servers. Each of the document servers is a HTTP (Hyper Text Transfer Protocol) server.

Figure 1 presents the web server cluster architecture. In this architecture, the load balancer has a single, virtual IP (Internet Protocol) address and request routing among servers is transparent. Every request from the clients is delivered into the load balancer over the internet. After that, the load balancer redirects the request to a document server in a round-robin manner.

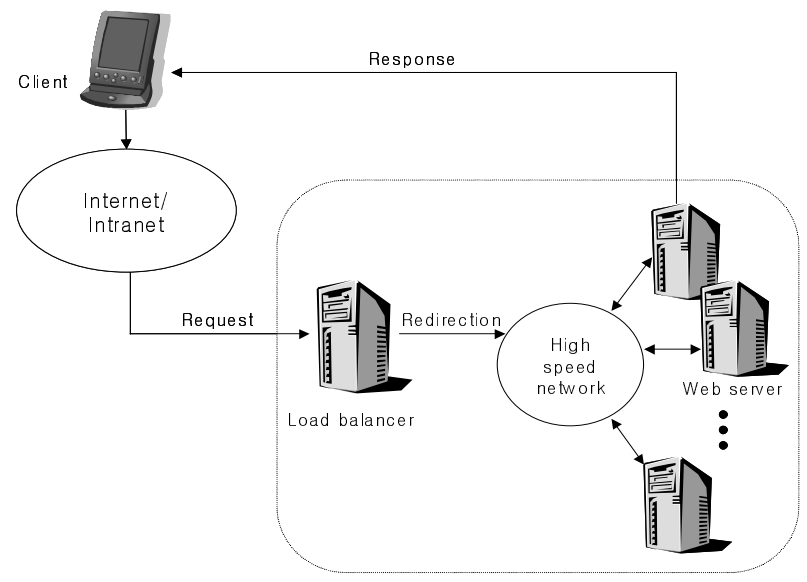

Fig. 1. Web server cluster architecture

Web documents that are serviced in the web server cluster are distributed on the disks of each node. Nodes that receive a user request are called the primary, and nodes that store the requested data on the disk are called the owner. This means that each node can be the primary or the owner as the case may be. The owner nodes maintain a directory in which they keep track of the copies of the data pages they own in global memory.

The only times a node has to be informed about status changes to the pages are when a page becomes a duplicate after being the only copy in global memory and when the page becomes the last copy in the node's memory after being a duplicate. 


\section{Memory Management of Web Server Cluster}

Efficient memory management is the task of keeping useful data closer to the user in the memory hierarchy. Figure 2 shows the memory hierarchy that is considered in this paper.

The user request is divided into page-sized units and is serviced by the primary node. If the requested page exists in the primary node, it is serviced immediately. If the page is absent, the primary node requests the owner node for the page. If the page presents in the owner node, it is forwarded to the primary node. Otherwise, the owner node forwards the request to the other node that has the requested page. The node receiving the forwarded request sends the data directly to the primary node. However, if no node contains the page, the request is satisfied by the owner's disk.

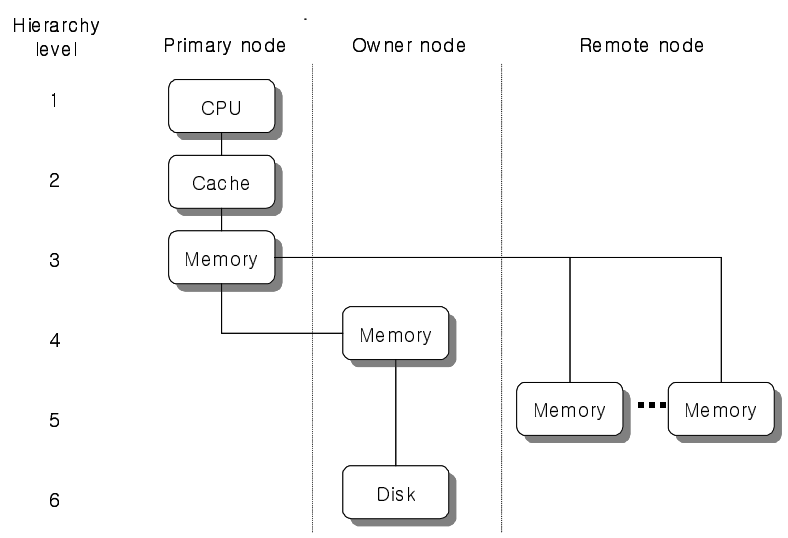

Fig. 2. Cluster memory hierarchy

When the owner node reads the page from disk, it retains a copy of the page in local memory. In order to improve global memory utilization, these pages are maintained on a FIFO (First In First Out) list and should be removed from the memory of the owner node as soon as possible.

The page replacement policy is to minimize the number of possible page faults so that effective memory access time can be reduced. The LRU algorithm is a popular policy and often results in a high hit ratio in a single server. This policy assumes that the costs of page faults are the same. However, this assumption is not valid in the cluster system, since the latency to fetch a page from disk and the latency to fetch a page from the remote node are different. Therefore, the elimination rate of the duplicated page in the global memory has to be higher than that of a single page.

Our proposed policy, DREAM (Duplication RatE Adjustment Method) for memory management of the web server cluster adjusts the duplication rate according to the various access distributions of web documents. The page replacement method of DREAM is as follows. 


$$
\begin{aligned}
& \mathrm{IF}(\mathrm{Ws} \times \mathrm{Cs}<\mathrm{Wd} \times \mathrm{Cd} \times \alpha) \\
& \text { Replace the LRU single page }
\end{aligned}
$$

ELSE

$$
\text { Replace the LRU duplicate page }
$$

Where $\mathrm{W}_{\mathrm{s}}$ and $\mathrm{Wd}$ are the inverse of the elapsed time since the time of last access for the LRU single page and LRU duplicate page, respectively. Cs is the latency to fetch a page back into memory from disk and $\mathrm{Cd}$ is the latency to fetch a page back into local memory from the remote memory. $\alpha$ is a parameter for duplication rate adjustment. $\mathrm{W}_{\mathrm{s}}, \mathrm{Wd}_{\mathrm{d}}, \mathrm{Cs}$ and $\mathrm{Cd}$ are positive and $\mathrm{Cd}_{\mathrm{d}}$ is lower than $\mathrm{Cs}$. Thus $\mathrm{Cs}_{\mathrm{s}} / \mathrm{Cd}$ is always higher than 1 .

In order to observe the impact of the parameter $\alpha$, let's consider the behavior of DREAM with $\alpha=0$. In this case, the ELSE statement is always performed since Ws $\times$ Cs is positive. This means that DREAM is Duplicate Elimination when $\alpha$ is equal to 0 . Also, when $\alpha$ is equal to 1 , DREAM is the same as the Hybrid algorithm.

If $\alpha$ becomes $\mathrm{Cs}_{\mathrm{s}} / \mathrm{Cd}_{\mathrm{d}}, \mathrm{W}_{\mathrm{s}}$ and $\mathrm{Wd}_{\mathrm{d}}$ are just compared at the IF statement. This means that DREAM is a Greedy Forwarding algorithm that only considers time information. Thus, we can see that Duplicate Elimination, Greedy Forwarding and Hybrid are special cases of DREAM.

When $\alpha<0$, the right term of the IF statement is negative. This case is the same to $\alpha$ of 0 . When $0<\alpha<1$, the elimination rate of the duplicated page is between Duplicate Elimination and Hybrid. When $1<\alpha<\mathrm{Cs}_{\mathrm{s}} / \mathrm{Cd}$, the elimination rate of the duplicated page is between Hybrid and Greedy Forwarding. Finally, when $\alpha>\mathrm{Cs}_{\mathrm{s}} /$ $\mathrm{Cd}$, there is no meaning since the elimination rate of a single page becomes higher than that of a duplicated page. Therefore, the valid range of parameter $\alpha$ is $0 \leq \alpha$ $\leq \mathrm{Cs} / \mathrm{Cd}$. Figure 3 shows the relation for the elimination rate of duplicated page and parameter $\alpha$.

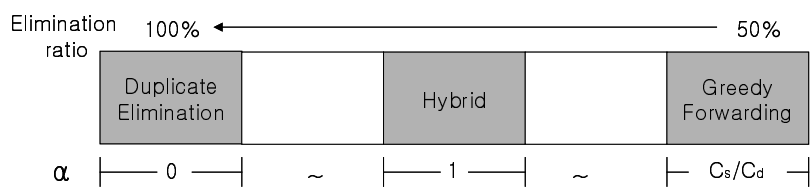

Fig. 3. The relation for the elimination rate of duplicated page and $\alpha$

In general, the access frequencies of web documents closely resemble a Zifpian distribution [10]. In this distribution, the shape of the curve is decided by the skew parameter. When skew is 0 the access frequencies of web documents are uniform, and when the skew parameter is 2 the access frequencies of web documents are skewed. 
The algorithm that has the best response time among Duplicate Elimination, Greedy Forwarding and Hybrid is decided by the skew parameter. DREAM has the best response time by adjusting parameter $\alpha$ according to the skew parameter. Also, it does not have an additional overhead for improving performance.

\section{Performance Evaluation}

As indicated by the title, this section is devoted to a description of the simulation results which we obtained using our memory management for web server cluster. Table 1 presents the simulation parameters and the Simscript II.5 process oriented simulation package is used to model the system.

Table 1. Simulation parameters

\begin{tabular}{|c|c|}
\hline Parameter & Value \\
\hline Number of nodes & 3 \\
\hline Memory per node & $64 \mathrm{MB}$ \\
\hline Number of files & 384 \\
\hline File size & $512 \mathrm{~KB}$ \\
\hline Page size & $8 \mathrm{~KB}$ \\
\hline Message cost & $1 \mathrm{~ms} / \mathrm{page}$ \\
\hline Network bandwidth & $15 \mathrm{MB} / \mathrm{sec}$ \\
\hline Disk bandwidth & $10 \mathrm{MB} / \mathrm{sec}$ \\
\hline
\end{tabular}

Figure 4 shows the response time as a function of skew. At low skew, Greedy Forwarding is worse than the other algorithms, but the response time decreases drastically as skew increases. To the contrary, Duplicate Elimination has a good response time at low skew, but the improvement of response time is little even if skew increases. Hybrid has a response time that is close to the minimum at both low and high skews, but it is not the best solution at all skews. DREAM eliminates duplicate pages in a similar way to Duplicate Elimination at low skew and it duplicates the hot pages in a similar way to Greedy Forwarding at high skew. Thus, it has always the best response time even though skew increases.

In Figure 5, the aggregate web document size that will be serviced is 2 times the size of global memory. In this case, Greedy Forwarding has the worst response time even though skew is high.

Also, the data size of Figure 6 is 0.5 times the size of global memory. In this case, Duplicate Elimination has the worst response time at every skew because each node has enough local memory. When the local memory is enough, the duplication of hot pages improves performance by eliminating network overhead. 


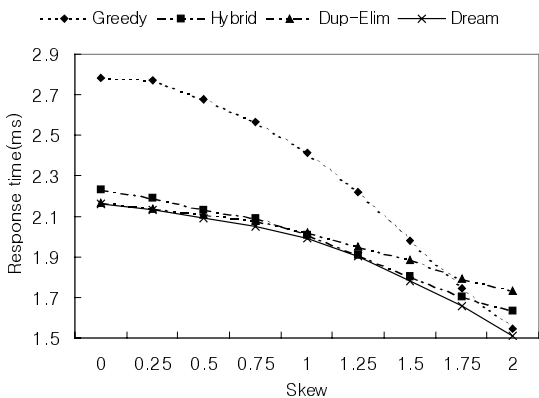

Fig. 4. Skew : Response time, (Number of files $=768$ )

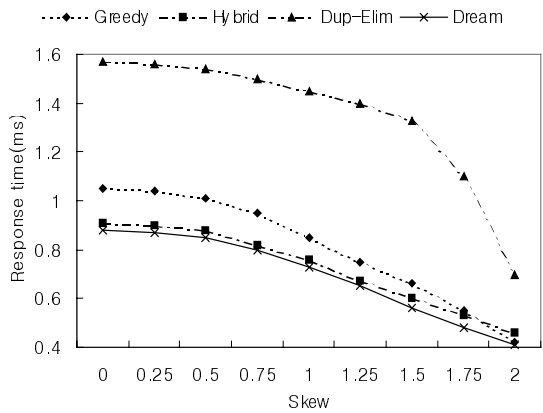

Fig. 6. Skew : Response time, $($ Number of files $=192)$

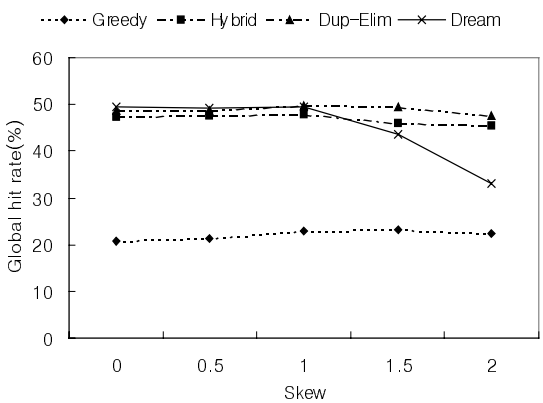

Fig. 8. Skew : Global hit rate

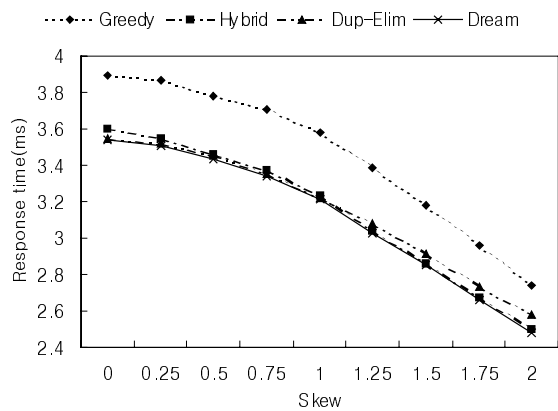

Fig. 5. Skew : Response time, (Number of files $=384$ )

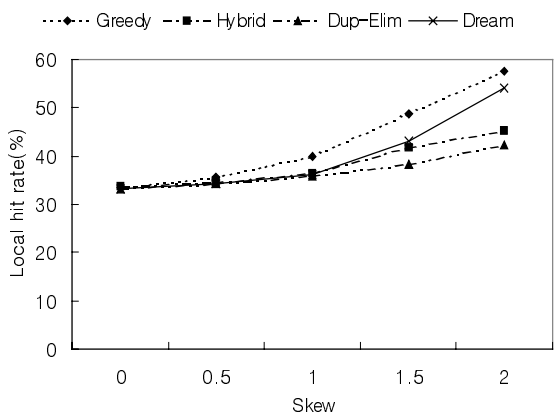

Fig. 7. Skew : Local hit rate

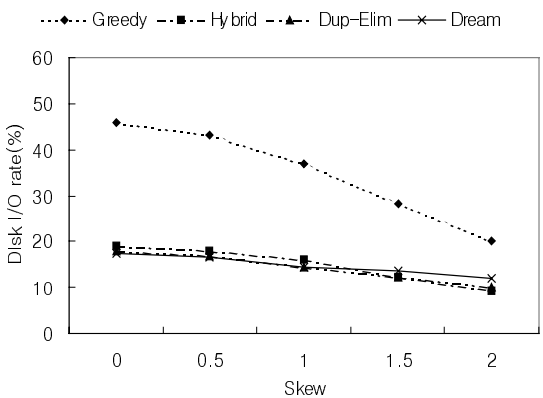

Fig. 9. Skew : Disk I/O rate 
Hybrid has a good response time on the average, but it does not have the minimum response time at all skews. On the contrary, we can see that DREAM has a best response time by adjusting $\alpha$ according to the skew.

Figure 7 and Figure 8 show the average local hit rate and global memory hit rate, respectively. In Figure 8, global hit rate of Greedy Forwarding is lower than other algorithms because disk I/O rate is high at the low skew and local hit rate is low at the high skew.

In addition, Figure 9 shows the disk I/O rate. Greedy Forwarding performs frequent disk access at the low skew but has the best local memory hit rate at the high skew. Duplicate Elimination has the worst local memory hit rate but has a good global memory hit rate. DERAM has the best response time by optimizing the ratio of the local memory hit rate, global memory hit rate and disk access rate.

\section{Conclusion}

Cluster systems are emerging as a viable architecture for building high performance and high availability servers in application areas such as web services or information systems. In initial cluster systems, processing power was a dominant factor of the performance, but memory bandwidth has replaced the role of the processor as a performance bottleneck. Thus, efficient memory management is very important for the overall cluster system performance. In this paper, we proposed an efficient memory management algorithm under the web server cluster. In addition, simulation results show that DREAM always achieves a good user response time for various skew parameters.

\section{References}

1. Du, X., Zhang, X.: Memory Hierarchy Considerations for Cost-effective Cluster Computing. IEEE Transactions on Computer (2000) 915-933

2. Cardellini, V., Colajanni, M., Yu, P.S.: Dynamic Load Balancing on Web-server Systems IEEE Internet Computing (1999) 28-39

3. Zhu, H., Yang, T., Zheng, Q., Watson, D., Ibarra, O.H., Smith, T.: Adaptive Load Sharing for Clustered Digital Library Servers. Proceedings of the Seventh IEEE International Symposium on High Performance Distributed Computing (1998) 28-31

4. Buyya, R.: High Performance Cluster Computing: Architectures and Systems. Prentice-Hall (1999)

5. Feeley, M. et. al.: Implementing Global Memory Management in a Workstation Cluster. In Proceedings of the 15th ACM SOSP (1995)

6. Venkataraman, S., Livny, M., Naughton, J.: Impact of Data Placement on Memory Management for Multi-Server OODBMS. In Proceedings of the 11th IEEE ICDE (1995)

7. Koussih, S., Acharya, A., Setia, S.: Dodo: A User-Level System for Exploiting Idle Memory in Workstation Clusters. 8th IEEE International Symposium on High Performance Distributed Computing (1999)

8. Dahlin, M., Wang, R., Anderson, T., Patterson. D.: Cooperative Caching: Using Remote Client Memory to Improve File System Performance. In Proceedings of the First Symposium on Operating Systems Design and Implementation (1994) 
9. Venkataraman, S., Livny, M., Naughton, J.: Memory Management for Scalable Web Data Servers. 13th International Conference on Data Engineering (1997)

10. Zipf, G.: Human Behavior and the Principle of Least Effort. Addison-Wesley (1949)

11. Guchi, M., and Kitsuregawa, M.: Using Available Remote Memory Dynamically for Parallel Data Mining Application on ATM-Connected PC Cluster. 14th International Parallel and Distributed Processing Symposium (2000) 